Pedagogía y Saberes n. ${ }^{\circ} 53$

Universidad Pedagógica Nacional

Facultad de Educación. 2020.pp. 11-19

\title{
El debilitamiento teórico de la pedagogía en la posmodernidad*
}

Artículo de investigación

The Theoretical Weakening of Pedagogy in Postmodernity Enfraquecimento Teórico da Pedagogía na Pós-Modernidade

Alejandro Álvarez Gallego**

Para citar este artículo:

Álvarez, A. (2020). El debilitamiento teórico de la pedagogía en la posmodernidad. Pedagogía y Saberes, 53, 11-19. https://doi. org/10.17227/pys.num53-10671

* Este artículo es producto del subproyecto de investigación “Educación popular, pedagogías críticas, estudios culturales y poscoloniales. Trayectos políticos y confluencias teóricas: Colombia, 1970-2008” que formó parte del proyecto de investigación interuniversitario "Paradigmas y conceptos de la educación en Colombia", aprobado por Colciencias con el código 1115-452-21145 bajo la coordinación de Jesús Alberto Echeverri, profesor de la Universidad de Antioquia.

** Doctor en Filosofía y Ciencias de la Educación por la Universidad Nacional de Educación a Distancia, Madrid, España. Profesor de la Universidad Pedagógica Nacional, Bogotá, Colombia. Grupo de Historia de la Práctica Pedagógica.

Correo electrónico: aalvarez@pedagogica.edu.co

Código Orcid: orcid.org/0000-0003-2537-4925. 


\title{
Resumen
}

Este artículo de reflexión es derivado del proyecto de investigación "Paradigmas de la pedagogía en Colombia: 1970-2000", financiado por Colciencias. El subproyecto de investigación que dirigí se ocupó de buscar los aportes de la educación popular al campo de la pedagogía. En el transcurso de la investigación nos encontramos con unos enunciados provenientes de la educación popular que coincidían casi literalmente con los discursos que agencian hoy las grandes corporaciones. Este hallazgo nos llevó a plantear que la pedagogía hoy también es producida en y para el capital. La pedagogía está enraizada en unos principios que en el pasado hacían parte de los discursos emancipadores que buscaban la formación de ciudadanos críticos capaces de cuestionar el sistema capitalista. Queda planteado el interrogante: ¿qué sería entonces hoy pensar la pedagogía?

\section{Palabras clave}

Pedagogía; posmodernidad; escuela; capitalismo

\begin{abstract}
This article derives from the "Pedagogical Paradigms in Colombia, 1970-2000" research project, which was sponsored by Colciencias. The research subproject I led aimed at researching the contributions of popular education in Colombia to pedagogy. Through the development of the research we found statements derived from popular education that closely matched current statements of big corporations today. These finding led us to suggest that even today pedagogy is produced by and to support capitalism. Pedagogy is rooted in some principles that used to be frequent in emancipation discourses, which looked to educate critical citizens, capable of questioning capitalism system. Now we ask, what is the meaning of thinking about pedagogy today?
\end{abstract}

\section{Keywords}

Pedagogy; posmodernity; school; capitalism

\section{Resumo}

Este artigo deriva do Projeto de Pesquisa "Paradigmas da Pedagogia na Colômbia: 1970-2000", financiado por Colciencias. O subprojeto de pesquisa que dirigi preocupava-se pela procura das contribuições da Educação Popular na Colômbia para o campo da pedagogia. No decorrer da investigação, encontramos alguns enunciados provenientes da Educação Popular que quase literalmente coincidiam com os discursos que as grandes corporações promovem hoje. Essa constatação nos levou a afirmar que a pedagogia também é produzida hoje no e para o capital. A pedagogia está enraizada em alguns princípios que costumavan ser frequentes nos discursos de emancipação, que procuravam educar cidadãos críticos, capazes de questionar o sistema capitalista. A questão que permanece é: como seria hoje pensar a pedagogia?

\section{Palavras chave}

pedagogia; pós-modernidade; escola; capitalismo 


\section{Introducción}

Este artículo busca sugerir ideas que nos ayuden a entender qué está pasando hoy con los maestros, los formadores de maestros, la pedagogía, la educación, la escuela, los niños, niñas y jóvenes, las políticas educativas y con la sociedad en general, cuando parece ser una verdad incuestionable que ya la escuela no es la única institución que educa. Esta es una verdad de a puño que tiene consecuencias trascendentales sobre la sociedad, porque durante más de trescientos años (por lo menos doscientos en nuestros países latinoamericanos) las sociedades occidentales se han organizado de tal manera que

- Niños, niñas y jóvenes pasan sus primeros años de vida estudiando en la escuela. Allí aprenden las nociones básicas del conocimiento socialmente aceptado con las cuales se puede ejercer la ciudadanía y acceder a los bienes culturales y económicos que la sociedad distribuye. Hoy eso se consigue de muy diferentes maneras.

- Fue en ese contexto en el que se configuró la pedagogía como el saber que habría de orientar los modos de educación escolarizados, en todas sus variantes. Hoy existen muchos otros modos de educación que le piden a la pedagogía su orientación.

- La sociedad se ocupó de discutir, ajustar y variar de mil maneras las formas como este proceso se hacía universal, se hacía laico o confesional, integraba o no a todas las clases sociales, incluía contenidos patrióticos o civilizatorios, se le entregaba a profesionales de la pedagogía o a profesionales formados en las disciplinas, se regulaba la disciplina con castigos o con el diálogo, se controlaba su funcionamiento desde el Estado central o con autonomías provinciales, se financiaba exclusivamente con el erario público o se toleraba el cobro a los particulares, se evaluaba a los individuos o a las instituciones, se priorizaba la educación del alma, la mente o el cuerpo...

Hoy las discusiones sobre la educación se han descentrado y las que se refieren a la escuela son apenas un capítulo de ese amplio repertorio pedagógico que hoy ocupa otros espacios.

Todo lo anterior da cuenta de las condiciones sobre las cuales se había constituido un modo de ser de la pedagogía. Hoy tenemos que reconocer que la pedagogía es un asunto que desbordó a la escuela. Es más, hablamos de pedagogías, en plural, para dar a entender que los objetos de sus preguntas van más allá de las cuatro paredes que le dieron origen en la modernidad.

Vamos a mostrar cómo la llamada posmodernidad ha incidido en estos cambios. Por posmodernidad entendemos aquí lo que planteó Lyotard (1987) cuando señaló que desde finales del siglo xx estaba cambiando el modo como se legitiman las verdades que hasta entonces eran consideradas ciencias; llamó condición posmoderna a la forma como se legitima el saber en esta época, en la que la verdad ya no depende de la prueba científica, sino de su puesta en escena, es decir, de manera performativa, en la acción misma, y esto debido al modo como circula hoy el conocimiento, más allá de los formatos clásicos propios de la modernidad (universidad y escuela). Según este planteamiento, en este artículo suponemos que estamos viviendo una época que transita por la posmodernidad, y que los discursos que antes tenían la pretensión de ser disciplinas científicas, como la pedagogía, están siendo transformados de manera que desbordan sus cánones modernos para circular en formatos no convencionales, en este caso a la manera de las llamadas competencias, como se mostrará.

Además, vamos a mostrar que las pedagogías críticas han contribuido al debilitamiento del estatuto teórico de la pedagogía al entrar en relación con el capitalismo cognitivo, lo cual, paradójicamente, pone en cuestión la viabilidad de un proyecto como el de la educación popular, tal como se le reconoció en el pasado. Muchos de los enunciados propios de dicha educación popular ya no tienen el estatuto de verdad que tuvieron en otras décadas; sin embargo, se están configurando nuevas prácticas que, articuladas con otros discursos de borde procedentes de saberes alternos, están dibujando un nuevo escenario propicio para pensar hoy las resistencias en el campo conceptual de la pedagogía. Esas configuraciones nuevas están todavía difusas, pero revisando la historia de las relaciones entre la educación popular y la pedagogía, pueden surgir pistas para leer los rastros de nuevos movimientos pedagógicos. Este asunto lo dejaremos abierto; no lo desarrollaremos porque está fuera del alcance de este trabajo; lo que queremos es mostrar la forma como se ha debilitado el discurso pedagógico y las posibilidades que se abren para pensarnos de otra manera.

\section{Los nuevos escenarios educativos}

Hoy se habla con naturalidad de la sociedad educadora y de la ciudad educadora, incluso se empiezan a institucionalizar los dispositivos pedagógicos que se utilizan para educar a la población más allá de la 
escuela. Los discursos sobre pedagogía ciudadana y pedagogías urbanas están en pleno furor. Muchos de esos discursos tienen un tinte fuertemente disciplinador y en algunos casos parecen estrategias de control, antes que otra cosa.

Los debates actuales sobre el problema de la gubernamentalidad, biopolítica y sociedades de control que han planteado Foucault (2007) y Deleuze (1991), entre otros, están poniendo en evidencia que los cambios en la sociedad contemporánea transforman los mecanismos tradicionales de gobierno de la población, pasando de las políticas de disciplinamiento ejercidas a través de las instituciones que la modernidad había creado (hospital, cárcel, escuela, ejército), a las políticas de control ejercidas a través de la publicidad, los medios de comunicación, las tecnologías de la información, las políticas de evaluación, el libre mercado, las guerras y el miedo. ${ }^{1}$

En ese sentido, habría que examinar hasta dónde estas nuevas pedagogías urbanas y ciudadanas están o no contribuyendo al afinamiento de las tecnologías de control que buscan hacer más expeditas las formas de circulación y acumulación del capital en las nuevas formas de producción económica instaladas por el posfordismo. Con una precaución: evitando los análisis simplistas que ven solo dominación en las formas de comunicación y de educación que se están creando. Desde nuestra perspectiva, en las dinámicas de cambio acelerado en que vivimos, donde se desestructuran tan fácilmente los discursos y las instituciones convencionales, hay prácticas que pueden ser direccionadas en sentidos diferentes y eso genera alternativas a los intereses que pretenden controlar y dominar.

Ahora bien, esta nueva realidad en la que la educación ha desbordado la escuela, le ha exigido transformarse. Allí aparece una nueva tensión relacionada con el interés del Estado, los empresarios, los organismos internacionales, los maestros, las comunidades, los jóvenes y las organizaciones sociales, para tener el control de ella. En ese sentido, los actores sociales reivindican la democratización de las políticas públicas en educación; para incidir en su destino, reivindican la democracia escolar para que de verdad puedan participar en la toma de decisiones sobre la vida cotidiana de la escuela. También los empresarios y los organismos internacionales negocian con el Estado la posibilidad de incidir en la toma de decisiones que

1 Algunos autores, como Paolo Virno (2003), han mostrado cómo el miedo se ha constituido en una condición que identifica el modo de ser de la cultura contemporánea. Para otros es un dispositivo de control muy eficaz, intencionalmente promovido, como lo dejó ver Michael Moore en su famoso documental Bowling for Columbine (2002). favorezcan la eficiencia, la productividad, la competitividad y la innovación. Desde todos los sectores se le pide a la escuela que flexibilice sus estructuras y que se disponga a estar abierta a transformaciones permanentes, tal como rezan los discursos posmodernos, tanto de tendencia neoliberal como de tendencia democrática. Como resultado de estas presiones y de la incidencia de estos actores en la vida de la escuela, se han operado transformaciones importantes, legales y prácticas, sobre los métodos de enseñanza y los contenidos curriculares.

Las otras educaciones, las que suceden por fuera de la escuela, desde las comunitarias, las indígenas, las de las organizaciones sociales populares, hasta las empresariales y las telemáticas en todas sus variantes, están introduciendo prácticas pedagógicas igualmente flexibles, versátiles, móviles, cambiantes, buscando acomodarse a la velocidad de los cambios sociales y culturales de la contemporaneidad. Este afán por cambiar golpea las puertas de la escuela cada vez con más fuerza y está guiando los discursos pedagógicos contemporáneos, pues se ven impelidos a ocuparse de los otros modos de educación.

Si interrogamos este presente es para buscar las condiciones en que están apareciendo los nuevos modos de ser de la pedagogía, las condiciones en las que se ha venido generalizando el discurso sobre el cambio, la flexibilización y la innovación. Dicho discurso hay que examinarlo con cuidado, porque tiende a imponerse como un fenómeno inevitable, como signo de los tiempos, como una realidad incuestionable. De hecho, es muy sugestivo: la tradición crítica lo ha utilizado, los defensores de la modernidad también. Recordemos la frase de Marx "Todo lo sólido se desvanece en el aire", que Marshal Berman (1991) convirtió en un libro para mostrar cómo la modernidad occidental se enfrentó a la tradición y a las instituciones comunitarias con ese principio de la innovación y el cambio permanente.

En el contexto Marxista, cuando se hablaba de cambios, se diferenciaban los cuantitativos de los cualitativos. Los primeros eran propios de la sociedad capitalista misma; en ese sentido, las contradicciones en las relaciones de clase iban produciendo cambios en el modo de producción que se acumulaban hasta producir el salto cualitativo revolucionario, que daría lugar al socialismo y luego al comunismo. El cambio revolucionario era el cambio estructural por el que trabajaba el proletariado. En el contexto liberal el cambio era un imperativo. El progreso era impostergable, inaplazable e inevitable. 
Por eso hay que preguntarse, antes de aceptar hablar de innovación y cambio: ¿a qué innovación y a qué cambio se refieren?, ¿para qué cambiar y al servicio de qué intereses? Podríamos decir que el problema no es la flexibilidad, o el cambio en sí mismos, sino saber en qué medida sirven para acabar o para incrementar los procesos de fetichización que nos hace extranjeros de nosotros mismos, la enajenación que nos hace vulnerables a las modas o a los discursos hegemónicos, la marginalidad y la discriminación que nos hace sentir inferiores, o la pobreza que nos inhabilita para ejercer los derechos fundamentales en condiciones dignas. Por eso hay que preguntar: ¿Quién es el Otro a quien se le pide cambiar? ¿A quién hay que cambiar? ¿Quién cambia a quién?²

\section{Las implicaciones para la pedagogía}

Hasta acá hemos planteado que la escuela se creó en la modernidad para introducir un cambio cultural en la población, de tal manera que asumiera un modo de vida en el que nada permanecería estático. También hemos dicho que la escuela se vio desbordada en su misión transformadora y que la educación adquirió otras formas, utilizó otros medios y la pedagogía se vio impelida a pensar más allá de la escuela, encontrándose de nuevo con el discurso del cambio como un imperativo inevitable. En conclusión, la pedagogía ha servido, desde la escuela y más allá de ella, para conseguir los propósitos transformadores que necesitaba la sociedad moderna. La formación de un sujeto funcional y adaptado a las necesidades de los postulados modernos había sido hasta hace poco la tarea de la pedagogía. Esta tarea la había asumido el Estado. La educación de la ciudadanía era una de sus más sagradas funciones, desde la escuela o fuera de ella.

En la llamada sociedad posmoderna, el discurso del cambio que acompañaba la necesidad de transformar las subjetividades llamadas tradicionales se ha intensificado y ha adquirido nuevos matices. Para pensar la pedagogía en este contexto vale la pena detenernos un momento a explorar qué tan sustantivas son dichas intensificaciones.

Lo que hoy sucede es que está mutando sustancialmente esa idea de pedagogía que teníamos. La formación de sujetos se ha liberado del control estatal. La disputa por lo que conviene o no hacer en relación con los procesos de subjetivación ya no depende de agencias centralizadas o de instituciones burocrá-

2 Peter Sloterdijk (2012) ha mostrado magistralmente cómo funciona hoy ese afán compulsivo por cambiar. ticas con dispositivos formativos intencionalmente dirigidos y administrados en función de intereses ideológicos. El problema político de la educación ya no parece ser ideológico, porque no hay un aparato institucional que controle de manera hegemónica las directrices que orientan pedagógicamente los procesos formativos. La formación de sujetos parece ser que ya no acontece solo en el espacio de la escuela. Entonces, ¿dónde nos constituimos en sujetos hoy?, ¿a través de qué mecanismos?, ¿qué subjetividades se configuran en la sociedad de hoy?, ¿quién o quienes luchan por controlar esos procesos?, ¿cómo y dónde se disputa hoy el control de nuestras subjetividades?

Hay una tesis en discusión que dice que el modo de producción capitalista ha cambiado su naturaleza, a tal punto que ya no produce fundamentalmente bienes materiales, sino subjetividades para ser intercambiadas en el mercado. Tal tesis no está anunciando el triunfo definitivo del capital sobre el trabajo; al contrario, sus autores plantean que allí el capital se está exponiendo por primera vez al juego de la indeterminación, del azar y de la incertidumbre, con lo cual el juego de posibilidades para escapar de la fetichización, la enajenación y la discriminación se abre infinitamente. Queda por discutirse si esto es suficiente para escapar también de la pobreza.

Dichas tesis hablan del posfordismo (Mejía, 2011), del capitalismo cognitivo (Blondeau, 2004), del capitalismo cultural (Bhabha, 2002), o de multitud (Hardt y Negri, 2004), según el autor; pero todos coinciden en que cambió la forma de acumulación del capital y por lo tanto la manera de producir plusvalía; la fórmula marxista del valor ya no funcionaría.

Las consecuencias de estas tesis están por analizarse en los diferentes ámbitos de la vida social. Una de ellas es la relacionada con los actores que intervienen en la producción del valor que hace que las mercancías sean competitivas en el mercado. Hablan entonces del concepto de general intellect, que Marx introdujo en los Groundisse. Lo que para Marx era el intelecto general, esto es, el conocimiento social incorporado en las máquinas (capital fijo), como resultado del proceso social de producción, en el posfordismo habría cambiado. Dicho intelecto general se habría liberado de las máquinas y se habría convertido él mismo en el medio de producción. En consecuencia, lo que caracteriza el capitalismo contemporáneo es que los medios de producción son inmateriales, y en dicha condición se convertirían en un bien social, en un bien público. Ahora bien, a pesar de que son un bien público (porque es producido de manera anónima por la inteligencia general), los capitalistas lo usan para generar el plusvalor del 
cual se lucran, pues en sus manos generan las tasas de ganancia más altas, razón por la cual atraen a los principales inversionistas del mundo. A eso es lo que hacen referencia los asesores de gestión del empresariado posmoderno, o management, cuando señalan cosas como:

Hoy, la táctica más auténtica es hacer algo con la retroalimentación del cliente y escuchar sus sugerencias [...] No tenemos clientes tenemos creyentes [...] Necesitamos un equipo que tenga el mismo ADN de nuestros clientes [...] Hoy, la táctica más auténtica es hacer algo con el feedback del cliente [...] Detrás del diseño de un logotipo, un eslogan y las demás piezas comunicativas, se halla todo un proceso de estudio de mercado, de la compañía, de cada producto y de los valores que se buscan transmitir, que a veces son más importantes que el producto mismo. Moliné (experto en publicidad), cita el caso de Dove, que si bien eventualmente promociona una crema o un champú, lo que refuerza todo el tiempo con su publicidad es el concepto de cercanía e identificación con la mujer, y de comprensión de su naturaleza. (El Espectador, 6 de octubre del 2008, p. 44)

Una organización de aprendizaje: Las organizaciones necesitan aprender a medida que enfrentan diferentes procesos de crecimiento para adaptarlo a su funcionamiento [...] En América Latina la educación va en la vía equivocada [...] sobran abogados y faltan ingenieros. Porque la creación de riqueza en este siglo se hará por medio de la propiedad intelectual, a diferencia del siglo pasado en el que la manufactura mandaba la parada. [...] lo más importante en este siglo es aprender a aprender, porque ahora, debido a la cantidad de cambios, hay que aprender a desaprender constantemente. ... Hacen falta más innovadores y menos administradores del statu quo [...] Las organizaciones que aprenden prosperaron en los 90 con Peter Senge, cuyo resultado fue una visión de organización de empleados con capacidad de crear y transferir conocimiento. [...] Los procesos de aprendizaje incluyen la generación, recopilación, interpretación y diseminación de la información. Para que tenga máximo impacto, el conocimiento debe compartirse de forma sistemática y definida. El conocimiento se puede mover lateral o verticalmente. El ejemplo más conocido de este enfoque es el proceso de evaluación después de la acción (AAR, After Action Review) del Ejército de Estados Unidos, que hoy se usa en muchas empresas, y que es una entrega de información sistemática después de cada misión. (El Espectador, 22 de septiembre del 2008, p. 60)
En un entorno globalizado, lo importante es desarrollar la habilidad para emprender negocios. La educación requiere un replanteamiento estratégico, que lleve a decisiones concretas. [...] El mundo de los negocios no puede ignorar que una persona con inclinaciones culturales o facilidad para los idiomas también es un empresario en potencia. (El Espectador, 21 de septiembre del 2008, p. 64)

Siempre que sea posible los gerentes deben organizar a los empleados en equipos autogerenciados, en los cuales tienen la autoridad sobre temas como control de calidad, programación y métodos de trabajo. Los empleados son una rica fuente de información sobre cómo hacer un trabajo y cómo hacerlo mejor [...] Los gerentes que operan con un estilo participativo cosechan enormes recompensas en eficiencia y calidad de trabajo. Los gerentes participativos dan a sus empleados libertad para operar y hacer cambios por su cuenta, basados en su conocimiento y experiencia. (Harvard Business School Publishing, 2008, p. 214).

[...] usar las tecnologías de punta para canalizar la inteligencia de las masas, para generar nuevas ideas, modelos de negocios, canales, etc. Ya se ha demostrado que si se unen las inteligencias de los empleados de una empresa, seguramente van a salir a la luz novedosas iniciativas. [...] Cisco lanzó un software de colaboración que permite a los emprendedores e innovadores de todo el mundo el desarrollo de nuevas ideas para que Cisco decida si las adopta y premie a sus proponentes. [...] Empoderar a las masas requiere un cambio de mentalidad en los niveles ejecutivos. [...] Pues llegó el momento de aceptar y acoger la inteligencia colectiva. (Guillermo Santos, El Tiempo, 21 de abril del 2008, p. 3).

Los educadores deben hacerle caso al consejo que los líderes les dan para robustecer la formación de los futuros empresarios del país: hay que mejorar la cultura general de los jóvenes. Es indispensable comprender que la cultura no es un adorno ni una "costura"; es el medio ideal para enriquecer la creatividad, que es la más poderosa herramienta con la que se construye la competitividad de una nación. (El Espectador, 28 de julio del 2008, p. 34).

Todos estos planteamientos traen consecuencias para el discurso pedagógico contemporáneo por varias razones. Primero, porque lo que importaría ahora sería formar en un conjunto de competencias básicas que le permita a la población mejorar las condiciones en las que se produce el conocimiento que se necesita para incorporarles valor a las mercancías y así lograr una economía competitiva en el mercado internacional. 
Segundo, la producción de bienes materiales ya no le interesa prioritariamente al capitalismo. Puede dejarle esa tarea a la iniciativa individual, local, asociativa, comunitaria, a la pequeña y mediana empresa. Esa pequeña empresa estaría manejada por emprendedores competitivos que tendrían que disputarse en el mercado los nichos locales de consumo. Las grandes empresas (las corporaciones de hoy en día, que no tienen un dueño fijo, sino accionistas volátiles) ensamblan esos bienes materiales y les incorporan el valor agregado que proviene del intelecto general y que es la marca, el sello, el logo. Dicho logo o mejor, marca, es un mensaje que educa. Con ella venden un modo de vida, un modo de ser, y producen una nueva subjetividad. Por eso los medios de comunicación en todas sus variantes telemáticas, reguladas por la imagen y la música, resultan estratégicos, y tales corporaciones las controlan. Para eso basta ver unos pocos ejemplos de los eslóganes que identifican a grandes marcas: "Se vale todo lo que eres" (ropa interior); "Vive rápido" (ropa informal); "El fin de lo convencional" (automóviles); "Cambia con el mundo" (ropa para hombres); "Nada es imposible, no existen límites, solo nuevos caminos" (calzado).

Lo que venden las grandes marcas es una manera de vivir, caracterizada por el riesgo, por la velocidad, la intrepidez, la osadía, la capacidad de romper con todo lo convencional, sin límites, vivir en la intemperie, expuestos al cambio permanente, a partir de lo que cada individuo desee.

Podríamos decir que allí la pedagogía vuelve a estar en el centro de los proyectos societales, pues es el discurso que da cuenta de la manera como se producen los sujetos, los modos de subjetivación propios de una sociedad. En los dispositivos telemáticos contemporáneos estarían las claves para entender cómo se producen hoy los sujetos, qué tipos de sujetos y a través de qué mecanismos. Allí se construye el discurso pedagógico contemporáneo.

Esos sujetos que produce el modo de ser del capitalismo actual son volátiles, múltiples, cambiantes, flexibles, creativos, transformadores de su realidad y la de su entorno, porque ese es el consumidor ideal y porque ese es el que mejor puede aportar al conocimiento que hoy necesita el capital para aumentar sus tasas de ganancia. A su vez ese es el individuo que está en mejor disposición de consumir los bienes inmateriales que se generan a través de los medios de producción contemporáneos, esto es: la industria cultural (música, video juegos, moda, cocina, turismo, estética corporal, deportes, espectáculos, software, etc.), más conocida como economía naranja.

\section{Preguntas abiertas}

Si aceptamos que el capitalismo de hoy produce fundamentalmente sujetos, subjetividades, sensibilidades, ideas, modos de ser, modos de pensar, ¿podría decirse entonces que el capitalismo es hoy un proyecto pedagógico?

La consecuencia de lo que hemos planteado hasta ahora es que la relación clásica entre pedagogía y política se ha transformado. Esto, porque el modo de producción depende hoy de las cualidades que le eran propias a la política (Virno, 2003), esto es, el virtuosismo, la improvisación, la capacidad de crear. Entonces el trabajo de hoy es político. Según Virno, habría por eso una saturación de la política. Lo público - el escenario de la política antes- es hoy el mundo del trabajo. El trabajo se realiza en el escenario de lo público que antes ocupaba la política. El trabajo ocupó la esfera pública y la política se volvió negocio, en tanto que la esfera pública donde actúa fue cooptada por el nuevo modo de producción posfordista. Hacer política hoy en día es parte del espectáculo mediático que produce también un plusvalor.

Antes, el mundo del lenguaje, de la comunicación y de la cultura eran esferas independientes de la economía. El marxismo había denunciado que la lógica performativa del capital estaba regulando el mundo de la cultura, y en ese sentido deshumanizaba la sociedad; pero según Virno, lo que hoy sucede es que la lógica de la comunicación, del lenguaje y de la cultura regula la producción económica y de ella depende el capital.

De esa manera, las grandes corporaciones hoy en día son escenarios pedagógicos en la medida en que se ocupan de la producción de subjetividades. Los nuevos pedagogos serían los creativos, los diseñadores, los publicistas de esas corporaciones. Su arte está, no solo en tener las competencias suficientes para innovar todo el tiempo y vender la novedad que requiere el mercado, esto es, los nuevos modos de ser de los sujetos ciudadanos contemporáneos, sino la capacidad para leer del intelecto general aquello que es más innovador, para capturarlo y mejorar los procesos de producción, al tiempo que se responde a las expectativas de las masas, ofreciéndoles los productos empaquetados en formatos de ideas que quieren consumir el público ávido de emociones fuertes y siempre distintas, exclusivas, diferentes.

El problema ya no es, entonces, la tecnificación de los procesos que regulan la vida social, la absorción del mundo de la vida por parte de la lógica económica (crítica de los fenomenólogos) o la deshumanización de la cultura (crítica de la Escuela de Francfort), o 
seguir creyendo que la explotación hoy se da en el trabajo asalariado (en la producción industrial de bienes materiales). El problema es entender cómo la lógica de la cultura regula la producción económica. Según estas lógicas, la pedagogía ya no realiza su compromiso político incluyendo a las masas trabajadoras en la escuela para formar un sujeto emancipado, como se lo propuso la sociedad moderna. Primero, porque la población ya no es analfabeta en su gran mayoría (problema que sí había ocupado a Gramsci y a Freire), y segundo, porque la alfabetización de hoy exige manejar otros códigos que no se limitan a la lectoescritura.

Las masas trabajadoras tampoco son asalariadas. Son las clases bajas y medias ya escolarizadas, desempleadas, subempleadas, o emprendedores con su microempresa, con las competencias básicas incorporadas: ciudadanas (participativos y disciplinados), laborales (capaces de producir por sus propios medios sus ingresos), lingüísticas (capaces de decodificar los lenguajes mediáticos), científicas (observadores y analíticos).

Si hoy hay masas analfabetas, tampoco son asalariadas; pero habría que saber que la educación de esas masas le interesa muchísimo al gran capital, para formar las competencias básicas que necesita el mercado. Por eso al gran capital le interesa sobre manera la escuela básica, para formar en la nueva ciudadanía capaz de consumir las subjetividades que venden y de producir las ideas que necesita para innovar y garantizar su competitividad. Pero no la escuela que disciplina, sino la que forma en competencias básicas.

Si es cierto que el capitalismo contemporáneo es un gran escenario pedagógico, entonces habría que replantear la relación entre pedagogía y política, porque estaríamos diciendo que ya no hacemos pedagogía para crear conciencia política, sino que la pedagogía, esto es, la producción de sujetos, es el modo como el capitalismo produce hoy sus ganancias. Estamos, como ya dijimos, de nuevo en el centro del problema. Las masas trabajadoras que producen hoy el valor agregado del que vive el capital, son los nuevos pedagogos, y la política es parte del performance del mercado contemporáneo.

Es en estas condiciones que el estatuto teórico de la pedagogía se debilita, toda vez que se pierde la relación clásica que planteaba la ciencia moderna, de donde proviene toda teoría: la relación sujeto-objeto. Ya no habría un sujeto que produce una reflexión sobre la práctica, ya no habría un proceso sistemático de recolección de información sobre el cual se puedan derivar leyes que permitan validar un modelo o un método para enseñar. La pedagogía ya no sería ese gran corpus de postulados sobre lo que es conveniente hacer para que los individuos se formen de acuerdo con unos valores o unos saberes necesarios, según lo que prescribía el Estado. Las pretensiones de la llamada pedagogía sistemática se verían fuertemente debilitadas, toda vez que la pedagogía ahora sería una experiencia de subjetivación cuyo valor depende de la subjetividad del individuo, y no de postulados validados por una comunidad científica.

Entonces volvamos a nuestra pregunta central:

- ¿Es posible, en estas condiciones, rescatar el ideal emancipatorio de la pedagogía?

- ¿Qué significa formar conciencia crítica, esto es, para el cambio, para la transformación, cuando las competencias que necesita el capital, ser innovador y creativo, son tan parecidas a las que pregonaba la teoría crítica?

- ¿Qué papel desempeñan el maestro crítico y la escuela alternativa en este nuevo modo de ser de la sociedad capitalista?

- ¿Si ya no hay una clase trabajadora asalariada, cuál sería el sujeto que se emancipa hoy?

- ¿Qué posibilidades tiene la pedagogía de disputarle al mercado ese proyecto de formación de sujetos que le es tan vital? Esto es, ¿podemos existir al margen del mercado?

Si algo debe reevaluarse hoy, no es tanto la pedagogía, la escuela o los aprendizajes o los maestros, porque todo eso ya está reevaluado por la posmodernidad posfordista, sino el sentido ético y político de lo que hacemos.

\section{Referencias}

Berman, M. (1991). Todo lo sólido se desvanece en el aire. La experiencia de la modernidad (5. - ed.). Siglo Xxi Editores.

Bhabha, H. (2002). El lugar de la cultura. Manantial.

Blondeau, O., Dyer Whiteford, N., Vercellone, C., Corsani, A., Rullani, E., Moulier Boutang, Y. y Lazzarato, M. (2004). Capitalismo cognitivo, propiedad intelectual y creación colectiva (trad., E. Rodríguez López, B. Baltza y A. García Pérez-Cejuela). Traficante de Sueños.

Deleuze, G. (1991). Posdata sobre las sociedades de control. En C. Ferrer, El lenguaje libertario (trad., M. Caparros, pp. 114-121). Nordan.

Foucault, M. (2007). El nacimiento de la biopolítica. Buenos Aires: Akal.. 
Hardt, M. y Negri, A. (2004). Multitud. Guerra y democracia en la era del Imperio. Madrid: Debate.

Lyotard, J (1987). La condición posmoderna. Madrid: Cátedra.

Mejía, M. R. (2011). La(s) escuela(s) de la(s) globalización(es) II. Entre el uso técnico instrumental y las educacomuicaciones. Bogotá: Ediciones Desde Abajo.

Sloterdijk, P. (2012). Has de cambiar tu vida. Sobre antropotécnica. Madrid: Pre-Textos.

Virno, P. (2003). Gramática de la multitud. Madrid: Traficante de Sueños.

\section{Noticias de periódico}

Santos, G. (21 de abril del 2008). El Tiempo, sección editorial.

[Sección opinión]. (28 de julio del 2008). El Espectador.

[Sección responsabilidad social]. (21 de septiembre del 2008). El Espectador.
[Sección educación]. (22 de septiembre del 2008). El Espectador.

[Sección económica]. (6 de octubre del 2008) El Espectador.

Harvard Business School Publishing (3 de noviembre del 2008). Revista Avianca, 44. 